\title{
MATHEMATICAL MODELING USING SEMANTIC NETWORKS FOR TEACHING
}

\author{
Manuel Gonzalez Hernandez, PhD \\ Oleksandr Karelin, PhD \\ Anna Tarasenko, PhD
}

Autonomous University of Hidalgo State, Institute of Basic Sciences and

Engineering, Academic Area of Mathematics

\begin{abstract}
The purpose of this paper is to show an alternative to teach how people can do mathematical modeling of natural sciences such as physics, biology, earth science, meteorology and engineering disciplines like computer science, artificial intelligence, but also social sciences such as economics, psychology, sociology and political science using semantic networks in order to get a graphical view of the relationships between the components of the problem to be modeled. The main idea is to use the semantic networks to perform the connection between the components involved in the modeling with the concepts, operators and all the possible characteristics of the objects in the processes'events in a fragment of reality for the problem being solved. Mathematical modeling using semantic networks should be apparently more obvious in the sense that the relationship of different components of the system to the model will be more evident. Semantic networks can help better to understand how the modeling of a physical phenomenon of engineering or other knowledge domain, it can be carry out more easily, because the semantic network clearly describes the relationships of objects and concepts of the phenomenon or problem to be modeling.
\end{abstract}

Keywords: Mathematical Modeling, Semantic Networks, Dynamical Systems.

\section{Introduction}

In this paper We present an alternative to teach how can be modeled some phenomena of areas like natural sciences and social sciences using semantic networks, our purpose is to show how people can teach mathematical modeling using semantic networks to get a graphical view of the relationships between the components of the systems to be modeled. 
A mathematical model is a description of a system using mathematical concepts and language. The process of developing a mathematical model is termed mathematical modeling. Mathematical models are used not only in the natural sciences such as physics, biology, earth science, meteorology and engineering disciplines e.g. computer science, artificial intelligence, but also in the social sciences such as economics, psychology, sociology and political science; physicists, engineers, statisticians, operations research analysts and economists use mathematical models most extensively. A model may help to explain a system and to study the effects of different components, and to make predictions about behavior, and simulate the system with it.

In the literature about this theme, we found several documents in treating semantic networks to know how to represent concepts or procedures in mathematics and also to showing paths in a semantic network to select the best way to understand how to solve some mathematical problems in the low level education, mainly to see how people should reasoning and apply some kind of mathematical operation. Mingzhen Li[Mingzhen, 2011]apply path analysis method analyzing the influential factors, on mathematical modeling academic achievement of high school students, toexplores the relationships among the influential factors in mathematical modeling academic achievement, those factors are: mathematical modeling self-monitoring ability, creativity level, the cognitive structure of mathematics and science subjects, mathematical modeling emotion, creative inclination, cognitive style and mathematical modeling belief and they got significant positive correlation with mathematical modeling.

Mathematical modeling refers to modeling learner ability to achieve success which includes carrying on the plan, inspecting, evaluating, providing feedback, making adjustment and controlling the whole mathematical modeling activity. Self-monitoring is the key element of metacognition, and its level may significantly influence the possibility and efficiency of success in problem solving [Li, 1997, 2007].

We thought that the semantic networks should be help and give an alternative to understand how those mathematical models can build them up. The advantage of these topics is to understand how the semantic network components such as the physical laws, assumptions, operators, data, and so on, are required to build a model and how those elements are related each other.

Mathematical models can take many forms, including but not limited to dynamical systems, statistical models, differential equations, or game theoretic models. These and other types of models can overlap, with a given model involving a variety of abstract structures. In general, mathematical models may include logical models, as far as logic is taken as a part of 
mathematics. In many cases, the quality of a scientific field depends on how well the mathematical models developed on the theoretical side agree with results of repeatable experiments. Lack of agreement between theoretical mathematical models and experimental measurements often leads to important advances as better theories are developed.

Mathematical modeling involves the construction of the model through the creation of real-world scenarios to identify the problem to be modeled making correct assumptions, collecting data and propose a tentative model, test assumptions, refine the model if necessary, adjust the model if appropriate data and analyzing the mathematical structure of the model to the sensitivity of the conclusions appropriate when the assumptions are not exactly found.

To find the mathematical model [Clive, 1980], [Frank, 2003], [kapur, 2005] not only for physical phenomena, but also social sciences or any other, we have to take into account the application of physical laws to specific systems or include unknown parameters that must be evaluated by tests. Sometimes however, the physical laws that govern the behavior of a system are not fully defined and formulate a mathematical model may be impossible. If so, an experimental model of the process can be used. In this process the system is subject to a set of known inputs and the extent of its starts, then the mathematical model is derived from the input and output relationships.

The main idea of this paper is to use semantic networks, in order to get a more illustrative way to introduce to mathematical modeling of physical phenomena or social sciences applications. Also semantic networks may be serves to find a reverse process, i.e. the question ihow we get a semantic network having a mathematical model in such application area?. This problem is of great interest but it will not be treated here. It is important to talk about the description of physical phenomena or problem in hand using natural language [Bobrow, 1968]. Clearly, semantic networks can help us to understand the modeling of physical phenomenon of engineering or other knowledge domain because it describes the relationships of objects, concepts of the phenomenon or problem in hand involved in the modeling, because people can explain the interrelation of the objects and concepts using Natural Languages to figure it out the semantic network.

Semantic networks ware used by Simons [1973], Shapiro [1971], Rumelhart and Norman[1972] and Schank [1973] have proved to be very serviceable structures for the encoding of knowledge, offering such recognized advantages as a convenient bidirectional linkage between semantically related data and an inherent facility for bundling deep conceptual case systems. However, in their conventional form, networks are rather clumsy constructs for representation of quantification. This clumsiness 
is largely a result of the difficulties encountered in specifying the scopes of variables.

Mathematical modeling using semantic networks should be most clear in the sense that it shows us the relationship between of the different components of the system, also the concepts, physical laws and principles involved in the description of the phenomenon or problem to be modeled, because it is feasible to keep track on graphs of the semantic network that it might have better understand.

The paper has been written, initiating with knowledge representation that includes abstraction of what we mean by a fragment of reality, some definitions of semantic networks and mathematical modeling. We gave a couple of examples that generated a system of linear algebraic equations and a differential equation of second order, conclusions and references.

\section{Knowledge Representation}

Given a fragment of reality and a physical phenomenon of engineering or other kind of problemto bemodeled, we can propose the following: if $\boldsymbol{P}$ is a set of sentences in natural language [Bobrow, 1977.1979] that describes such phenomenon and if the corresponding set $\boldsymbol{K}$ of well-formed formulas coming from $\boldsymbol{P}$, as a result of natural language transcription [Bobrow, 1975] [Winograd, 1972, 1975] using an intermediate formal language like calculus of predicate of first order, then, there is a semantic network of $\boldsymbol{P}$ that correspond to the problem in hand.

This proposition leads to a series of consequences for their validity. In the literature there are separate results, such as having a semantic network into predicate calculus for every well-formed formula to describe situations of events that are described in natural language, mainly for representing knowledge in a context and that is one of the schemes knowledge representation in Expert Systems of Artificial Intelligence area, however from the point of view of mathematical modeling we did not find something similar to modeling dynamic systems using semantic networks. We have to mention the research technique from Henry Paynter on graphs link (Bond Graphs) [Paynter, 1961]. Bond Graphs concept was originated in 1961 by Henry Paynter, many investigators subsequently joined to Henry Paynter's idea like Karnoppeg, Rosenberg, Margolis D. L. [Karnopp and Rosenberg, 1968, 1975, 1983, 1990], [Margolis, 1990] Cellier [Cellier, 1991] etc.. The Bond Graphs are a domain-independent graphical description of the dynamic behavior of physical systems. This means that systems of different domains (electrical, mechanical, hydraulic, thermodynamic, acoustic etc.) are described in the same way. The Bond Graphs are based on energy and its interchanges. Professor H. M. Paynter of MIT invented the procedure; 
however it is not possible to model other kind of problems with Bond Graphs without energy concept.

The use of mathematical modeling mainly consists in replacing the cognitive object with its mathematical image i.e. its mathematical model, which implemented by logical and numeric algorithms through computers, allows studying the main patterns of the actual process. Such a cognitive method shows the advantages of theoretical and empirical approaches. Working with the mathematical model instead of focusing into the cognitive object, in a fast and cheap manner, permits to analyze and forecast state properties theoretically. At the same time numeric methods allow, using the computer calculation power, through numeric experiments, to verify its behavior and patterns not easily reached by the analytical approach.

Now, if we have to build a mathematical model of some physical phenomenon of engineering within a fragment of reality, we will describe it using natural language and with this description get a set of sentences in natural language which must be grammatically correct. The description of the processes and events using objects, concepts, operators, etc. of that fragment of reality, required to building the mathematical model of the problem in hand, so that, this set of sentences can be transcribedin a set of well-formedformulas using the first order predicate calculus.

In a fragment of reality where there are objects, events, concepts, processes, etc. that occur there and describes the possible data structures thereof, to propose a model of that piece of reality, bringing together all the above in a set of sentences in a formal language, we make the following analysis: Let $\boldsymbol{M}$ be aset of eventsofsome fragmentof reality and $\boldsymbol{R}$ a set ofprocesses of objectsand structuresof $\boldsymbol{M}$ that allowan interpretation.Let $\boldsymbol{m}$ be theset of images of $\boldsymbol{M}$, i.e.images thatcan be taken fromeventsthat occur ina logical wayto concludea process, soit should be understoodthat there is alogical formalismof relationsof $\boldsymbol{M}$ and $\boldsymbol{m}$.

Let $\boldsymbol{r}$ bea set of processesanddefinitions in $\boldsymbol{m}$ than allow a representing ofthe eventsof $\boldsymbol{M}$, so $\boldsymbol{r}$ is saidto be arepresentation scheme. It is definedasa body of knowledgeof the structure $(\boldsymbol{M}, \boldsymbol{R})$ or $(\boldsymbol{m}, \boldsymbol{r})$. The possibility of interpreting of the elements of $\boldsymbol{r}$ as events $\boldsymbol{E}_{1}, \boldsymbol{E}_{2}, \ldots, \boldsymbol{E}_{\boldsymbol{n}}$ ensures that there is a transformation $\boldsymbol{f}(\boldsymbol{M}, \boldsymbol{R}) \rightarrow(\boldsymbol{m}, \boldsymbol{r})$, and this representation is completein the sense that there is for each event $\boldsymbol{E}_{\boldsymbol{i}} \mathrm{of} \boldsymbol{M}$ an image $\boldsymbol{m}_{\boldsymbol{i}} \mathrm{of} \boldsymbol{m}$. That is,let $\boldsymbol{m}$ be a set of all possible images of $\boldsymbol{M}$. In this context we mean an image as a modelof an event $\boldsymbol{E}_{\boldsymbol{i}} \mathrm{of} \boldsymbol{M}$. The relationship between the imagesof the elements ensures their existence, thereby obtaining a structure called image's structure. More specifically, if $\boldsymbol{E}_{\boldsymbol{i}}$ is an event of $\boldsymbol{M}$ and $\boldsymbol{m}_{\boldsymbol{i}}$ an image of $\boldsymbol{m}$ from $\boldsymbol{E}_{\boldsymbol{i}}$, we can think of the structure of image $\boldsymbol{m}_{\boldsymbol{i}}$ as the relation coordinate between image and reality, and it ensures that $\boldsymbol{m}_{\boldsymbol{i}}$ is a complete 
representation of $\boldsymbol{E}_{\boldsymbol{i}}$, this is called a reproductive relation or of reproduction. Reproductive relationship allows the representation of the existing properties of the objects and the relationships between them in the event $\boldsymbol{E}_{\boldsymbol{i}}$ of $\boldsymbol{M}$. Let $\boldsymbol{f}$ be the reproduction function that relates an event $\boldsymbol{E}_{\boldsymbol{i}}$ of $\boldsymbol{M}$ with image $\boldsymbol{m}_{\boldsymbol{i}}$ of $\boldsymbol{m}$. The reproduction allows us represent those properties of the relationships between elements of $\boldsymbol{E}_{\boldsymbol{i}}$ that are not preserved in $\boldsymbol{m}_{\boldsymbol{i}}$ but must be represented explicitly.

Let $\boldsymbol{P}$ be the set of all feasible natural language sentences in this fragment of reality in which belongs $\boldsymbol{M}$. If the proposition $\boldsymbol{P}_{\boldsymbol{i}}$ of $\boldsymbol{P}$ is an image $\boldsymbol{m}_{\boldsymbol{i}}$ of $\boldsymbol{E}_{\boldsymbol{i}}$ in $\boldsymbol{M}$, then all that a proposition communicates about an event $\boldsymbol{E}_{\boldsymbol{i}}$ of $\boldsymbol{M}$ is its reproductive relation, this is because each object carries a name and the relationship between them is an event $\boldsymbol{E}_{\boldsymbol{i}} \mathrm{of} \boldsymbol{M}$. It is important to note that a proposition without proper grammatical structure, not represent an image $\boldsymbol{m}_{\boldsymbol{i}} \mathrm{of} \boldsymbol{E}_{\boldsymbol{i}}$ in $\boldsymbol{M}$. Theform of reproductionis necessary foran image $\boldsymbol{m}_{\boldsymbol{i}} \mathrm{of} \boldsymbol{m}$ or a proposition $\boldsymbol{P}_{\boldsymbol{i}}$ of $\boldsymbol{P}$ isa complete representation ofan event $\boldsymbol{E}_{\boldsymbol{i}}$ of $\boldsymbol{M}$.It can say thatall descriptions ofan event $\boldsymbol{E}_{\boldsymbol{i}}$ thatisin $\boldsymbol{M}$, isobtained from $\boldsymbol{m}$. Thus $\boldsymbol{P}$ and $\boldsymbol{m}$ mustbelogically equivalent.That is, we want tohavethe possibility of referringto $\boldsymbol{r}$ eitherthroughpropositions of $\boldsymbol{P}$ or throughimages $\boldsymbol{m}$ or both.Thus we haveobtainedanevent $\boldsymbol{E}_{\boldsymbol{i}}$ of $\boldsymbol{M}$ that can be describedthrough a proposition $\boldsymbol{P}_{\boldsymbol{i}}$ from $\boldsymbol{P}$.

The engineeringphysical phenomenain the reality fragment can be described by means of elementsof $\boldsymbol{P}$. Let $\boldsymbol{K}$ be the set ofwell-formed formulas ofelements of $\boldsymbol{P}$ whichdescribes theengineeringphysical system. Since eachelementof $\boldsymbol{K}$ has an associatedsemantic network,thenthe combinationof themgives usthe semantic networkofthe entire physical system.

Withthe idea of elucidatebetter theengineeringsystems modeling, we give some simples concepts onsemantic networksand their relation tothe formal languagepredicate calculusof first order.

\section{Semantic Networks}

Semantic networks were treated in Aristotelian philosophical era by the Greek philosopher Porphyry in his commentary on Aristotle's categories as a way to illustrate the methods of Aristotle to define categories.

Basically, it can distinguish six types of semantic networks [John F. Sowa,2008] and those types can see in the Encyclopedia of Artificial Intelligence, edited by Stuart C. Shapiro, [Shapiro , 1987].

A semantic network or net is a graph structure for representing knowledge in patterns of interconnected nodes and arcs. Computer implementations of semantic networks were first developed for artificial intelligence and machine translation, but earlier versions have long been used in philosophy, psychology, and linguistics. The Giant Global Graph of 
the Semantic Web is a large semantic network find in Berners-Lee et al. [Berners-Lee, 2008]; and Hendlerand van Harmelen in [Hendler, 2008].

What is common to all semantic networks is a declarative graphic representation that can be used to represent knowledge and support automated systems for reasoning about the knowledge. Some versions are highly informal, but others are formally defined systems of logic.

The first formalized knowledge representation were given by Quillian, R. [Quillian, 1968], Raphael, B. [Raphael, 1968] and Shapiro andWoddmansee [Shapiro, 1971]. The semantic network schemes have a very solid psychological foundation.

In the literature of this subject, there are several documents that treat the theme mainly related with the knowledge and basically with the social sciences like psychology for example, some networks were explicitly designed to implement hypotheses about human cognitive mechanisms, while others have been designed primarily for computer efficiency. Sometimes, computational issues may lead to the same conclusions as psychological evidence. The distinction between definition and affirmation networks, for example, has a close parallel to Tulving'sdistinction between semantic memory and episodic memory[Tulving, 1972].

Network notations and linear notations are capable of expressing equivalent information. But certain kinds of information are easier to express or process in one way or the other. Since the boundary lines are vague, it is impossible to state necessary and sufficient conditions that include all semantic networks while excluding other systems that are not usually called semantic networks.

The most common networks known in several papers on artificial intelligence, philosophical, psychological and linguistic area, are:

Networks, IS-A: In which the links between nodes are labeled. Conceptual graphs: In which there are two types of nodes, concepts and relationships.

Frames: In which the junction points of the links are part of the node's label.

In general, when we speak about semantic networks [Shastri, 1988] we often referred to one of these schemes, typically IS-A networks or framebased schemes, which share certain fundamental characteristics, ofthese shared characteristics include default inheritance.

The frame-based schemes allow great flexibilityand they have received the most attention from researchers in cognitive science and linguistics [Simmons, 1973]. Definitely the kind of semantic networks are IS-A quintessential.

In fact a semantic network is used when one has knowledge that is best understood as a set of concepts that are related to one another.Most 
semantic networks are cognitively based. They are intractable for large domains. Finally they don't represent performance or meta-knowledge very well.Some properties are not easily expressed using a semantic network, e.g., negation, disjunction, and general non-taxonomic knowledge. Expressing these relationships requires workarounds, such as having complementary predicates and using specialized procedures to check for them, but this can be regarded as less elegant.

The networks IS-A are the results of the observation that much of human knowledge is based on the assignment of a subset of items as part of a more general one. The natural classical taxonomies are a good example: Mass-Spring (MS) is a Dynamical System (DS), A Dynamical System is a combination of components (Comb_Compts), A combination of components is a physical system. We can express those sentences in a symbolic form using calculusof predicate of first order, to get a set of well-formed formulas (wff); those wffshave an implicitly semantic network.

$(\forall x)(M S(x) \rightarrow D S(x))$

$(\forall x)(D S(x) \rightarrow$ Comb_Compts $(x))$

$(\forall x)\left(C o m b \_C o m p t s(x) \rightarrow\right.$ Physical_System $\left.(x)\right)$

The conceptual graphs, proposed byJohnSowa[Sowa 1984] differ from IS-A networks inthat the arcsare not labeled,and the nodesare of two types: conceptnodes, which can represent bothan entityas a stateor process and relationnodes, which indicate how they relate totheconceptnodes. Therefore there arerelatednodesthat makethe role oflinksbetween entities.

There are two conceptual graphs notations, the linear form, and the unfolded form or diagrams, the notation types of elements are given by: The conceptual nodes drawing by rectangles, the relationship nodes drawing by circles, the arrows that give the flow of direction to the nodes, and the operation nodes drawing by circles within them an operator. For example,figures 1 and 2.

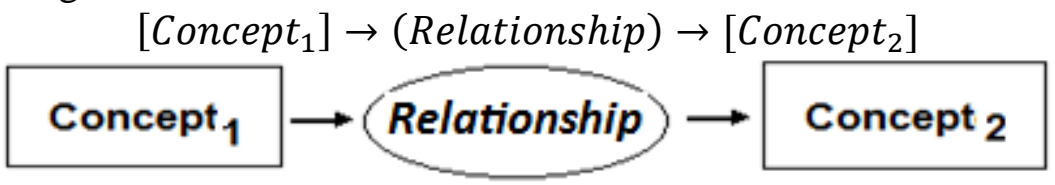

Figure 1. Notations of conceptual graphs

$\left[\right.$ Quantity $\left._{1}\right] \rightarrow$ (Operator $) \rightarrow\left[\right.$ Quantity $\left._{2}\right]$

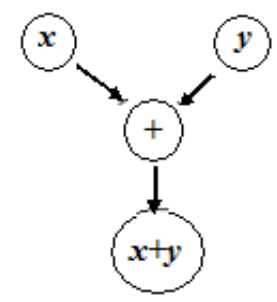

Figure 2. Notations ofoperational graphs 
In this way wecan be generatedgraphs that represent conceptsquite complex. As an example, we present one for thedynamical systemmassspring.

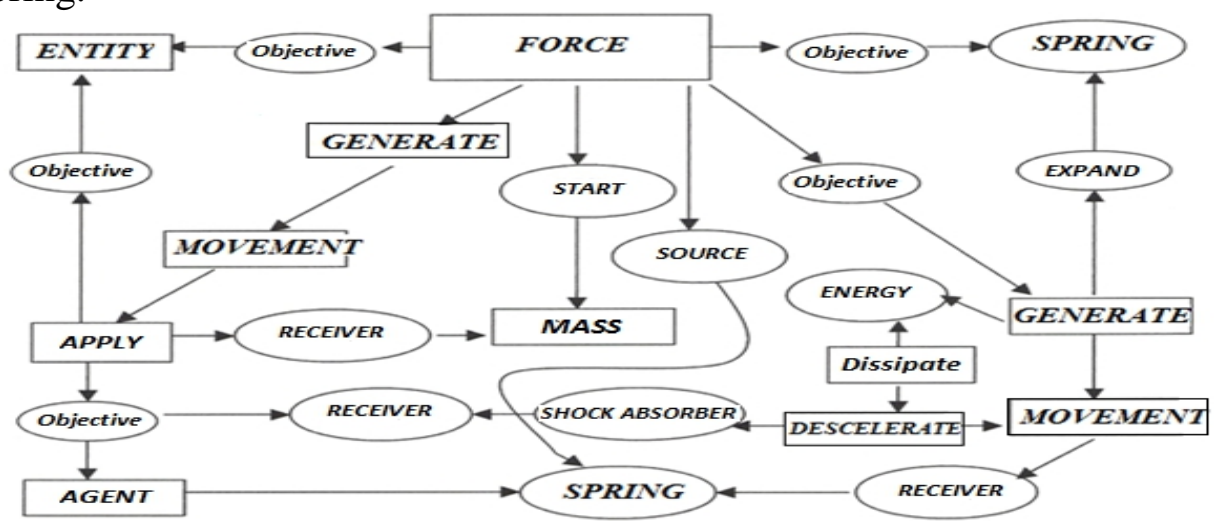

Figure 3ConceptualGraph for the dynamical system ForceMass-Spring.

In the semantic network of figure 3, the mass, which is the receptor for applying spring force and the agent, is the recipient of movement. The Force is an entity whose goal is to apply motion. The spring is the instrument of force, which generates movement to expanding, the energy generated by this fact is dissipated due to the shock absorber and decelerate the movement. The source of force is the spring that is the agent of application's force and the shock absorber as the receiver.

In a semantic network, the information is expressed by a set of nodes connected to each other by a set of labeled arcs, showing the relationships between them. One sentence in natural language is a symbolic representation in predicate calculus of the first order and in turn has a graph description. From the formal point of view, the terms, symbolic constants and variables and functional expressions are represented by the nodes of the graph and predicates by arches, the tail of the arc leaving the node represents the first argument of the predicate and the head of the arc entering to the node represents the second argument, for example the sentence "All springshave potential energy", in the predicate calculus of first order is represented as:

$$
\text { has (SPRING, POTENCIAL_ENERGY) }
$$

orlike:

$$
(\forall x)(\operatorname{SPRING}(x) \rightarrow \text { POTENCIAL_ENERGY }(x))
$$

Where the argumentsin this case aresymbolic constants, representedin upper case, itsassociated graphof this example is:

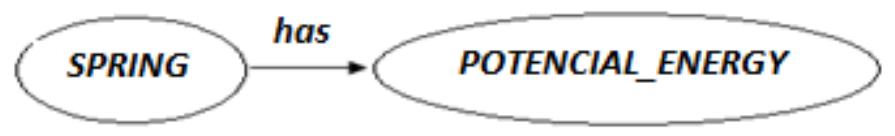


The nodesand arcsof thesegraphs arelabeled bythe termsof the predicatethat theydenote.

The semantic network sare useful to descry bepurposes that are givenin astructural graph ofset of facts. The networknodesare labeled bysymbolic constants,alsoit can be labeledwith variablesthatwill bealphabet's lowercase, for example, $x, y, z \ldots$ the variables arestandardizedandare assumedseparatelyuniversallyquantified.The scope ofsuch quantificationis the set offactsonthe entire semantic network.

In order to represent graphically therelationships ofpossible componentsof a dynamic system or other kind of problem that we want to model knowing that a system is a combination of components that work together to achieve a specific objective, we will seehow to obtainthe semantic networkassociated with it.

Not limited to physical systems, the concept of a system can be extended to abstract dynamic phenomena, such as those found in economic, transportation, population growth, biological, etc.

Any attempt to design a system should start with a prediction of execution before the system itself can be designed in detail or actually built. This prediction is based on the mathematical description of the dynamic characteristics of the system. This mathematical description is called a mathematical model. For most physical systems mathematical models are described in terms of differential equations.

The purpose of presenting the modeling of the system using a semantic network is to make more explicit the relationship of each component and how the concepts are involved and its content for people that want to teach how builds a mathematical modeling. The central idea is to establish a way to elucidate the mathematical model and interpret the interactions of dynamic system components, primarily identifying the inputs and outputs thereof.

\section{Mathematical Modeling}

Mathematical modeling involves the construction of the model through the creation of real-world scenarios to identify the problem to be modeled making correct assumptions, collecting data and propose a tentative model, test assumptions, refine the model if necessary, adjust the model if appropriate data and analyzing the mathematical structure of the model to the sensitivity of the conclusions appropriate when the assumptions are not exactly found. We illustrate the modeling process in the following figure4. 


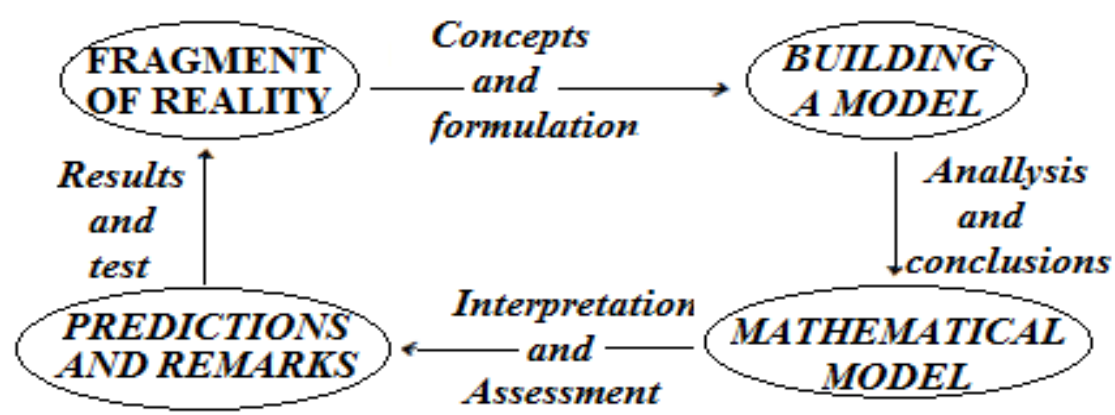

Figure 4. The usual process to get a mathematical model

Example: We would find the mathematical model, of the following problem:

"Betty and Laurita have some stamps, between the two are eighty, three times Betty's has five more than twice of Laurita has".

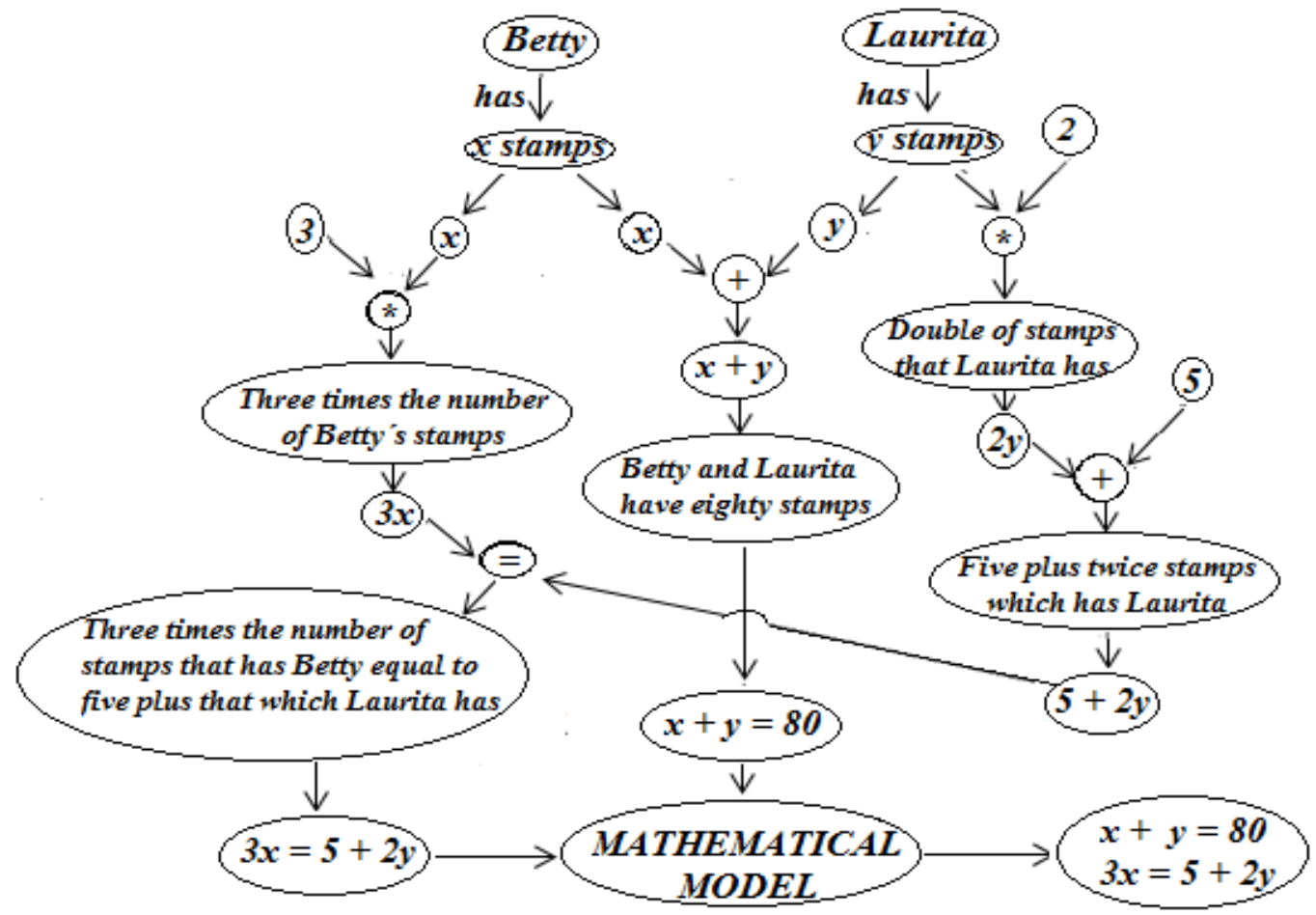

Figure 5 Semantic network of the above example gives us a linearsystem ofequations.

Taking each sentence of the text of the above problem we can obtain a set of well-formed formulas and build up the semantic network as we show in figure 5 .

The next proposition gives a description of a dynamical systemthrough the semantic network. 
Proposition 1. Let $\boldsymbol{S}$ be a dynamical system taken from a fragment of reality $\boldsymbol{M}$ and $\boldsymbol{R}$ the set of events that occur in $\boldsymbol{S}$, let $\boldsymbol{P}$ be the set of sentences in natural language that describes each of the events of $\boldsymbol{S}$ belong to $\boldsymbol{R}$ and their relationships, and let $\boldsymbol{K}$ be the set of well-formed formulas obtained from each element of the set $\boldsymbol{P}$. Then there exists a semantic network induced by $\boldsymbol{P}$ that describes the dynamical system $\boldsymbol{S}$.

As an example of the above proposition, let's take the dynamical system mass-spring as the system $\boldsymbol{S}$, then all the components and its relationships, we can describe them using natural language and build the set $\boldsymbol{P}$ that contains all the sentences that describe $\boldsymbol{S}$, therefore exist a set $\boldsymbol{K}$ of well-formed formulas (wff).

If $\boldsymbol{S}$ is a dynamic system, and $\boldsymbol{P}$ a set of natural language sentences describing $\boldsymbol{S}$, then exist a set of well-formed formulas obtained from $\boldsymbol{P}$, since every well-formed formula has associated a semantic network, and because the wffs are constituted of atomic formulas, then the network's nodes also are atomic, i.e. when they correspond to these atoms not change and if there is a set of well-formed formulas, then there exist a semantic network to be constituted of all networks generated for each wff. Therefore we can say that the proposition 1 gives the feasibility that carry out this formulation to get a mathematical model.

To illustrate themass-spring dynamic systemmodeling, we start withthe diagramof Figure 6, which consists of a mass $\boldsymbol{m}$, damperandspring, is a system withone degree of freedom, and it is an examplein whichthe oscillationscan be describedwithjust one coordinateq(t).

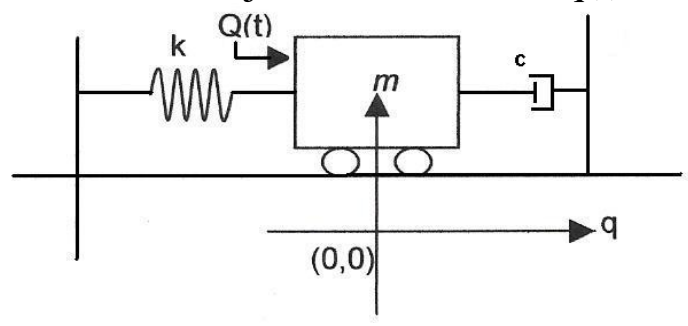

Figure 6.The dynamicmass-springsystemwithdamping.

To illustrate those facts consider the following sentences obtained from Figure 6. They are facts and propositions that elucidated the massspring system.

"If the mass moves, the $q(t)$ is increased, then the spring exerts a force $f_{s}$ on the mass such as the force is equal to - $\mathrm{kq}(\mathrm{t})^{\text {“. }}$. Which can be written in terms of predicates like:

$$
\begin{gathered}
\text { moves }(M A S A, I N C R E A S E-q(t)) \\
\text { exercises }\left(F O R C E-f_{S}, M A S S\right)
\end{gathered}
$$

And graphing as 


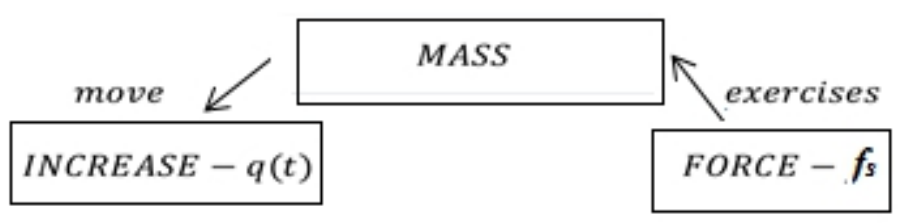

Figure 7.The Semantics Network generated by the natural language sentences.

Now using semantic networks we can elucidate the mathematical model, with a better view on the relationship of system components, as shown in Figure 8. That maybe we described using natural language as follow.

The system consists of a mass $\boldsymbol{m}$ which can it slide along a horizontal plain. The mass acts on a spring of constant $k$, a damper with coefficient $c$ and an external force $Q(t)$. The position of the mass at any time during its movement it is specified by the coordinate $\mathrm{q}(\mathrm{t})$ measured from its mass's equilibrium position. The force $Q(t)$ it's acting in one directiontending toincrease $q(t)$. When the massmoves a distance $q(t)$ from itsequilibrium, the spring exerts a force $f_{s}=-k q(t)$ and also the damper exerts a force $f_{d}=-c \frac{d q(t)}{d t}$ on the massm. Applying Newton's second law of motion, we equate the forces acting on the mass, for its acceleration, obtaining: Netforce $=f_{s}+f_{d}+m a$, then, $m \frac{d^{2} q(t)}{d t^{2}}=Q(t)-k q(t)-c \frac{d q(t) \text { therefore }}{d t}$ motion's equation is given by: $m \frac{d^{2} q(t)}{d t^{2}}+c \frac{d q(t)}{d t}+k q(t)=Q(t)$ or using dot operator $\quad \cdot=\frac{d}{d t}$ looks like this $m \ddot{q}+c \dot{q}+k q=Q(t)$ which is the mathematical model of the physical phenomenon described, as we show in figure 8 involving all those elements that we were talking about of the semantic networks, maybe you aware that we didn't give set $\boldsymbol{P}$ of sentences and the set $\boldsymbol{K}$ for the well-formed formulas (wffs).

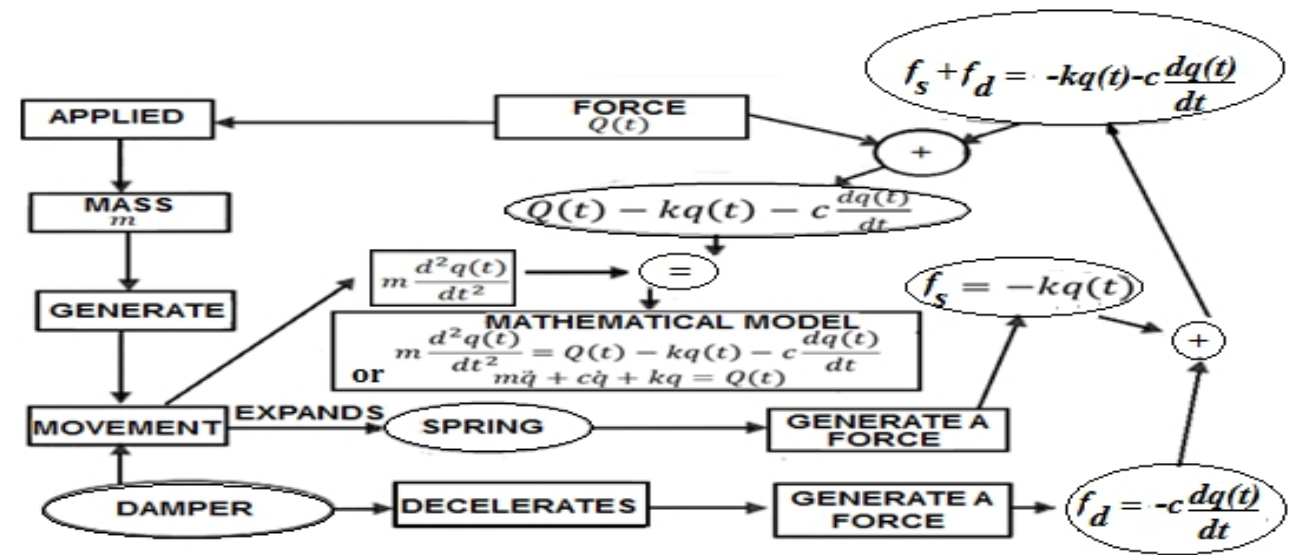

Figure 8. The semantic network for the dynamical system mass-spring to getting the mathematical model for it 


\section{Conclusion}

Any attempt of teaching mathematics has an incentive mainly knowing that all the effort served for any person can improved their learning in mathematical modeling and to find alternatives for this purpose. Mathematical modeling is one of the most salient issues on applied mathematics because of the need to test and verify the results of experiments through simulation using the mathematical model and especially for those experiments that are too expensive or maybe not feasible to repeat, due to the impossibility in its magnitude in the physical phenomenon or both, as in the various parameters involved on it, for example the events that usually occur in a nuclear power plant. We should mention that it was interesting tracking the sequence of steps to build the mathematical model, following trail in the fragment of reality that can it help to understand how to build the mathematical model using semantic networks. Finally we can say that using semantic networks to teach mathematics it will be more comprehensible the process to build the mathematical model especially for those who are beginners in this area.

\section{References:}

Bobrow, D. G. \& T. Winograd "An Overview of KRL, a Knowledge Representation Language", Cognitive Science 1: 3-46. 1977

Bobrow and A. Collins(eds.) Representation and Understanding. New York: Academic Press.1975

Bobrow, D. G."Natural Language Input for a Computer Problem-solving System", en M. MinskyB.(ed.) Semantic Information Processing. Cambridge, Mass: MIT Press, 146-226.1968.

Bobrow, D. G. and T. Winograd "KRL. Another Perspective", Cognitive Science 3: 29-42.1979

Bobrow, D. G. "Natural Language Input for a Computer Problem-solving System", en M. MinskyB.(ed.) Semantic Information Processing. Cambridge, Mass: MIT Press, 146-226.1968

Bouma, G. "Feature Structures and Nonmonotonicity", Computational Linguistics, Special Issue on Inheritance I, Vol. 18, No 2: 183-204.1992

Berners-Lee, Tim, James Hendler, andOraLassila.The Semantic Web,Scientific American, May 2001.

Cellier, F.E., Continuous System Modeling, Springer Verlag, ISBN 0387 975020

Clive L. Dym and Elizabeth S. Ivey.(1980) Principles of Mathematical Modeling.Academic Press.1991

Frank R. Giordano, Maurice D. Weir and William P.Fox.First Course in Mathematical Modelling. Thomson. 2003 
Genesereth, M. R. \& J. NilssonLogical Foundations of Artificial Intelligence. San Mateo, California: Morgan Kaufmann. 1987

Hendler, James A., and Frank van Harmelen The Semantic Web: webizing knowledge representation, in van Harmelen et al. pp. 821-839. 2008.

Joseph W. Jerome.Modelling and computation for Applications in Mathematics, Science, and Engineering.Clarendon Press Oxford.1998

Kapur, JagatNarain Mathematical Modeling. Ed.New Age International Publishers,2005.

Karnopp D.C., and Rosenberg R.C.Analysis and Simulation of Multiport Systems - The bond graph approach to physical system dynamics, MIT Press, Cambridge MA. University of Twente, Dept EE Intro Bond Graphs 31 / 31. 1968

Karnopp D.C., and Rosenberg R.C.System dynamics, a unified approach, J Wiley, New York, NY. 1975.

Karnopp D.C., Margolis D.L., and Rosenberg R.C.System dynamics, a unified approach,(2nd edition), J Wiley, New York, NY, ISBN 047145940 2. (Textbook, a teacher's guide isalso available). 1990.

$\mathrm{Li}$, M.Study on mathematical modeling cognition mechanism and teaching strategy. (Unpublished doctoral dissertation).Southwest University, Chongqing, China.2007

Minsky M.A Framework for Representing Knowledge.MIT-AI Laboratory Memo 306.1974.

Mingzhen Li, Qinhua Fang, ZhongCai, Xinbing Wang.A Study of Influential Factors in Mathematical Modeling of Academic Achievement of High School Students.Journal of Mathematics Education.June 2011, Vol. 4, No. 1, pp.31-44.2011

Paynter, H.M.Analysis and design of engineering systems, MIT Press, Cambridge, MA. 1961.

Rosenberg R.C., Karnopp D.C. Introduction to physical system dynamics, McGraw Hill, NewYork, NY. 1983

Quillian, R. "Semantic memory" in Semantic Information Processing, M. Minsky (Ed),MIT Press, Cambridge, Mass.,

Raphael, B. "A Computer Pogram for Semantic Information Retrieval” in Semantic Information Processing, M. Minsky (Ed),MIT Press, Cambridge, Mass. 1968.

Ritchie, G., S. Pulman, A. Black and G. Russell"A Computational Framework for Lexical Description", Computational Linguistics, Vol. 13, No. 3-4: 290-307.1987.

Shapiro, S.C. and G. H. Woddmansee."A Net Structured Based Relational Question-Answerer", Proceedings International Joint Conference on AI. Washington DC, 325-346. 1971. 
Shapiro S. C. Encyclopedia of Artificial Intelligence, edited by Stuart C. Shapiro, Wiley, 1987. 1987

Shastri, L.Semantic Networks: An Evidential Formalization and Its Connectionist Realization. Los Altos, California: Morgan Kaufmann. 1988 Simmons, R. F. "Semantic Networks: Their Computation and Use for Understanding English Sentences."Computer Models of Thought and Language.Ed. R. Schank and K. Colby. San Francisco: W. H. Freeman. 1973.

Sowa, John F. (2008)Conceptual graphs, in van Harmelen et al. pp. 213-237. 2008.

Sowa, J. F. Conceptual Structures: Information Processing in Mind and Machine. Reading, Mass: Addison-Wesley. 1984.

Tulving, Endel. Episodic and semantic memory, in E. Tulving\& W. Donaldson, eds., Organization of Memory, Academic Press, New York.1972. Winograd, T. (1972) Understanding Natural Language. New York: Academic Press.

Winograd, T. "Frames and the Procedural-Declarative Controversy", inD. G. 1975.Winston, P. H.Artificial Intelligence. Reading, Mass: Addison Wesley. 1977. 